Somatic complaints in early adulthood predict the developmental course of compassion into middle age

Saarinen, Aino I. L.

2020-04

Saarinen , A I L , Keltikangas-Järvinen , L, Lehtimäki , T, Jula , A , Cloninger , C R \& Hintsanen, M 2020 , ' Somatic complaints in early adulthood predict the developmental course of compassion into middle age ' , Journal of Psychosomatic Research , vol. 131 , 109942 . https://doi.org/10.1016/j.jpsychores.2020.109942

http://hdl.handle.net/10138/328921

https://doi.org/10.1016/j.jpsychores.2020.109942

cc_by_nc_nd

acceptedVersion

Downloaded from Helda, University of Helsinki institutional repository.

This is an electronic reprint of the original article.

This reprint may differ from the original in pagination and typographic detail.

Please cite the original version. 
This is the accepted manuscript of the article, which has been published in Journal of Psychosomatic Research. 2020, 131, 109942. https://doi.org/10.1016/j.jpsychores.2020.109942

\section{Somatic complaints in early adulthood predict the developmental course of compassion into middle age}

Running head: Somatic complaints and compassion

$$
\begin{aligned}
& \text { Aino Saarinen }^{1,2, *} \text {, Liisa Keltikangas-Järvinen }{ }^{2}, \text { Terho Lehtimäki }^{3} \text {, } \\
& \text { Antti Jula }{ }^{4}, \text { C. Robert Cloninger }{ }^{5}, \text { Mirka Hintsanen }^{1}
\end{aligned}
$$

${ }^{1}$ Research Unit of Psychology, University of Oulu, Finland

${ }^{2}$ Department of Psychology and Logopedics, Faculty of Medicine, University of Helsinki, Finland

${ }^{3}$ Department of Clinical Chemistry, Fimlab Laboratories and Faculty of Medicine and Health Technology, Finnish Cardiovascular Research Center, Tampere University, Tampere, Finland

${ }^{4}$ Department of Public Health Solutions, National Institute for Health and Welfare (THL), Helsinki, Finland

${ }^{5}$ Department of Psychiatry, Washington University, St. Louis, United States

* Corresponding author: Aino Saarinen. Department of Psychology and Logopedics, Faculty of Medicine, University of Helsinki, Helsinki, Finland. Postal address: Haartmaninkatu 3, P.O. Box 21, 00014 University of Helsinki, Finland. Email: aino.i.saarinen@helsinki.fi. Tel.: +358443071204. 


\begin{abstract}
Objective: The aim of the present study was to investigate (i) whether somatic complaints predict the developmental course of compassion in adulthood, and (ii) whether this association depends on alexithymic features. Methods: The participants came from the population-based Young Finns study ( $N=471-1037$ ). Somatic complaints (headache, stomachache, chest pain, backache, fatigue, exhaustion, dizziness, heartburn, heartbeat, and tension) were evaluated with a self-rating questionnaire in 1986 when participants were aged between 18-24 years. Compassion was assessed with the Compassion Scale of the Temperament and Character Inventory (TCI) in 1997, 2001, and 2012. The data were analyzed using growth curve models. Results: We obtained a significant compassion-age interaction $(\mathrm{B}=-0.137, p=0.02)$ and a compassion-age squared interaction interaction $(\mathrm{B}=0.007, p=0.006)$, when predicting the course of somatic complaints. Specifically, in participants without frequent somatic complaints, compassion steadily increased with age in adulthood. In participants with frequent somatic complaints, however, compassion remained at a lower level until the age of 40 years, then started to increase, and achieved the normal level of compassion approximately at the age of 50 years. The association between somatic complaints and compassion over age was found to be independent of alexithymic features. The analyses were adjusted for a variety of covariates (age, gender, use of health care in childhood, depression in childhood, parental socioeconomic factors, parental care-giving practices, stressful life events, parental alcohol intoxication, and participants' socioeconomic factors in adulthood). Conclusion: Frequent somatic complaints may predict delayed development of compassion in adulthood. This association was found to be independent of alexithymic features.
\end{abstract}

\title{
Keywords
}

Compassion; Personality; Somatic complaints; Physical symptoms; Somatization 
Somatic complaints and compassion

\section{Introduction}

Somatic complaints refer to physical symptoms (such as pain, dizziness, heartburn, heartbeat, or tension) that are authentic and cause suffering but appear not to be fully accounted for by a medical condition (1). Previous evidence has shown that individuals with frequent somatic complaints cause an enormous strain to the public health care system (2). For example, individuals with somatoform disorder utilize health care services substantially more frequently and cause approximately two times higher health care costs in both outpatient and inpatient clinics, when compared to an average citizen $(3,4)$. Individuals with somatic complaints are also found to have increased risk for health-related job loss (5) and prolonged sickness absences (6), even more than 120 days per year (3).

Somatic complaints are known to result from a complicated and reciprocal interplay between endogenous genetic and biological vulnerabilities, early adversities, sociocultural factors, and triggering environmental factors $(1,7,8)$. The most crucial biological vulnerabilities for somatic complaints include certain temperament dimensions. In particular, the temperament dimensions of inhibition, distractibility, intensity, and negative affectivity are linked to stronger pain responses, higher reactivity of sympathetic nervous system (including heart rate reactivity and skin conductance), and somatic complaints (9-11). In individuals with such biological temperament-related vulnerabilities, stressful situations or psychosocially adverse circumstances may trigger or intensify somatic complaints $(1,12,13)$, particularly when capacity for emotional self-regulation is weak because of immature character development $(14,15)$

Extensive prior work has shown that Somatization Disorder is positively associated with antisocial personality and alcohol use disorders both in individuals and in families $(7,8,16)$. Antisocial personality is characterized by low levels of TCI Cooperativeness, including increased hostility and decreased empathy, compassion, and moral reasoning $(17,18)$. However, whether one's suffering due 
to somatic complaints might predict one's compassion for others' suffering has not been investigated specifically, particularly not in longitudinal studies. Compassion for others is defined as a disposition to feel concern for other's suffering that is followed by the desire to alleviate the suffering and improve the other's well-being (19). Compassion can refer to compassionate states (temporary compassionate feelings for others that vary in line with situational factors) or dispositional compassion (a more stable trait that endures over time) (19).

Previous studies suggest that there may exist several potential pathways from frequent somatic complaints to less mature compassion development in adulthood. Firstly, there is a great amount of evidence that somatic complaints commonly co-occur with alexithymia (20-22).

Alexithymia primarily includes challenges in several affect- and cognition-related processes as follows: challenges in emotionalizing (reduction of emotional experiences), identifying (ability to define one's emotional states), analyzing (ability to explain one's emotional states), verbalizing (ability to describe or communicate one's emotional reactions), and fantasizing (reduction of fantasies or other inner processes) $(23,24)$. These challenges partly derive from neurocognitive impairments (e.g. attentional processes, speech processing) and neurobiological alterations (e.g. altered functioning of affect-related brain regions) (25). That is, individuals with somatization symptoms may not recognize somatic complaints as a manifestation of psychological distress but they may rather interpret somatic complaints as a marker of medical disease $(1,20)$. Abilities to recognize affective states from the face and body, in turn, are necessary requirements for experiencing compassion for other's suffering (19). There may exist also social pathways from frequent somatic complaints to lower level of compassion. Specifically, somatic complaints are found to commonly interfere with interpersonal relationships (1). It seems to be that frequent somatic complains are strongly related to both one's own less affiliative behavior toward others and, respectively, others' hostile reactions to individuals with somatic complaints. That is, by definition, somatization disorder refers to heightened attention and 
Somatic complaints and compassion

"excessive time and energy" directed to somatic complaints that may eventually dominate one's identity and life (1). This may likely interfere with directing attention to others' needs and having affiliative goals in social relationships that, in turn, are linked to less frequent experiences of compassion (26). On the other hand, somatic complaints (without a medical condition) are found to arouse weaker compassionate reactions from others, even hostile responses or willingness to take social distance in some cases $(27,4)$. Experiencing low social support from others, in turn, is related to lower levels of compassion for others (28).

Finally, previous evidence suggests that there may exist cognitive-affective pathways from somatic complaints to compassion. That is, somatic complaints are linked to catastrophic thinking processes: individuals with somatic complaints commonly experience feelings of threat and harm and have pessimistic expectations about the life (1). This may possibly interfere with mature compassion development since experiencing compassion includes the willingness to alleviate others' suffering (i.e. find solutions to suffering) and is linked to experiencing rather positive emotions (without sharing the negative emotions of the suffering other) (19). The catastrophic thinking processes may be further heightened along with anxiety and depressive disorders that are shown to be very common comorbid disorders in individuals with somatization disorder $(1,20,27)$.

The present study examined (i) whether somatic complaints predict the developmental course of compassion in adulthood between the ages of 29-50 years, and (ii) whether the association can be obtained independently of alexithymic features or whether it seems to proceed via alexithymic features. We used the Young Finns data with a 26-year prospective follow-up from the measurement of somatic complaints to compassion. In order to differentiate somatic complaints from clearly obtained medical conditions, accidents, depression, and stressful environment, we took into consideration a variety of potential confounders (use of health care due to somatic diseases or physical accidents; 
parental socioeconomic factors, parental care-giving, parental alcohol intoxication, and stressful life events; participants' socioeconomic factors and alexithymic features).

\section{Methods}

\subsection{Participants}

We used data from the prospective Young Finns Study. The participants were selected randomly from six age cohorts (born between 1962-1977) from the population register of the Social Insurance Institution. The Social Insurance Institution covers the whole population of Finland. The original sample included 3596 participants in the baseline measurement in 1980 (when participants were aged 3-18 years). The participants have been followed since then so that the latest follow-up measurement with compassion assessment was in 2012 (participants were aged 35-50 years). The study was carried out in accordance with the Declaration of Helsinki. Furthermore, the design of the Young Finns Study was approved by the ethical committees of all the Finnish universities with medical schools. Before participation, all the participants or their parents (for participants aged below 12 years) provided written informed consent after the nature of the procedures had been fully explained. The design of the Young Finns Study is described with more detail elsewhere (29).

For this study, family environment was evaluated in 1980; somatic complaints, depression, and use of health care in 1986; participants' socioeconomic factors and alexithymic features in 2011; and compassion in 1997, 2001, and 2012. The study design is illustrated in Supplementary Table 1. Only the two youngest age cohorts (born in 1974 and 1977) responded to the questionnaire of somatic complaints. In the analyses, we included all the participants with data available on the study variables. The final sample included 471-1037 participants in the analyses (i.e. $\mathrm{N}=1037$ in the baseline model; and $\mathrm{N}=471$ in the fully-adjusted model). 


\subsection{Measures}

\subsubsection{Somatic complaints}

Somatic complaints were assessed with a 10-item self-rating questionnaire asking how often the participant had experienced the following somatic complaints: headache, stomachache, chest pain, backache, fatigue, exhaustion, dizziness, heartburn, heartbeat, or tension. All the items were responded with a 4 -point scale ( $1=$ rarely or never; $2=$ once a month; $3=$ once a week; $4=$ daily). The internal reliability of the scale was good (Cronbach's $\alpha=.74$ in our study sample). All the items were further dichotomized into two categories $(1=$ once a week or more frequently; $0=$ once a month or less frequently). We calculated the mean score of the dichotomous items for all the participants who had responded to at least $50 \%$ of the items.

\subsubsection{Compassion}

Compassion was evaluated with Temperament and Character Inventory (TCI) (30). The compassion scale includes 10 self-rating statements (e.g., "It gives me pleasure to see my enemies suffer" [reverse scored], "It gives me pleasure to help others, even if they have treated me badly" [positively scored], "I like to imagine my enemies suffering" [reverse scored] and "I hate to see anyone suffer" [positively scored]). The items were answered using a 5-point Likert-scale ( $1=$ completely disagree; $5=$ completely agree). The reliability and validity of the Compassion Scale has been described thoroughly previously (31). In this study, the internal consistency of the scale was found to be high (Cronbach's $\alpha=.83-.86$ in 1997, 2001, and 2012). The mean score of compassion was calculated for all the participants with data on at least $50 \%$ of the items. The mean scores of compassion were standardized with the mean and standard deviation of year 1997 compassion scores, in order to stabilize the growth trajectories between different measurement years. 


\subsubsection{Psychosocial and socioeconomic family environment}

Psychosocial family environment consisted of 3 indicators: stressful life events, parental alcohol intoxication, and parental care-giving. Stressful life events included 7 events: moving residence $(0=$ no; $1=\mathrm{yes})$, change of school $(0=$ no; $1=$ yes $)$, parental divorce $(0=$ parents living together; $1=$ parents not living together $)$, mother's death $(0=$ no; $1=$ yes $)$, father's death $(0=$ no; $1=y e s)$, mother's hospitalization $(0=$ less than 10 days; $1=10$ days or more), father's hospitalization $(0=1$ ess than 10 days; $1=10$ days or more). We calculated a sum score of the stressful life events. Parental alcohol intoxication was evaluated by asking the frequency of parents' alcohol intoxication with a 8 -point scale $(1=$ never; $8=$ daily). We calculated the mean between the frequencies of mother' and father's alcohol intoxication. Parental care-giving was evaluated with a self-rating questionnaire filled by parents in 1980 . The questionnaire included 8 items that were rated with a 5-point scale. For example, "The child is significant to me" (1=very significant; 5=not significant) or "In difficult situations, the child is a burden" (1=totally disagree; 5=totally agree). This questionnaire has been used also previously (32). The internal consistency for the scale was high in the Young Finns data $(\alpha=0.70)$. The items were dichotomized to reflect hostility of parental care-giving: agreement with the item (scores $3-5)=1$; disagreement with the item (scores $1-2)=0$. We calculated a sum score of the dichotomous items.

Socioeconomic family environment included parents' level of income and educational level. Parental educational level was classified into 3 categories $(1=$ comprehensive school; $2=$ high school or occupational school; $3=$ academic level). In case mother's and father's educational level were different, we used the higher level of education. Level of parental income included 8 categories $(1=$ less than 15000 Finnish mark per year; 8=more than 100000 Finnish mark per year).

\subsubsection{Other covariates}


Somatic complaints and compassion

Other covariates included participants' use of hospital care (in 1986), depression (in 1986), alexithymic features (in 2011), and socioeconomic factors in adulthood (in 2011). Use of hospital care was evaluated by asking the participant in 1986 whether he/she had been in hospital care during the past six years $(1=$ no; $2=y e s)$ and whether the participant had visited doctor due to a physical accident $(1=$ no; 2=yes). Depression was evaluated in 1986 with one question by asking how often the participant feels depression (1=rarely or never; $4=$ daily).

Alexithymic features were evaluated with the Toronto Alexithymia Scale (TAS-20) (33). At the time of the study measurements, the TAS-20 had been used recently in another large Finnish study. Hence, the TAS-20 was adopted also in the Young Finns Study in order to provide a national reference and to make between-study comparisons. TAS-20 includes 20 items that are rated with a 5-point scale ( $1=$ strongly disagree; $5=$ strongly agree). The scale has been widely used also previously $(34,35)$. We calculated the mean score of the scale for all the participants with data available on at least $50 \%$ of the items (in practice, all of those participants had responded to at least $70 \%$ of the items).

Regarding psychometric properties of the scale, the convergent and discriminant validity and internal reliability of the scale have been demonstrated previously $(33,36)$. Also in our sample, the internal consistency of the scale was high (Cronbach's $\alpha=0.83$ ). Regarding factor structure, the findings have been slightly inconclusive, with some findings questioning the structural validity of the third factor $(36,37)$. In this study, we used the total score of the TAS-20 in order to avoid potential spurious results of some single subscales. Moreover, the predictive validity of the scale has been confirmed by demonstrating that high TAS-20 scores correlate with, for example, dissociative tendencies (38), posttraumatic disorder (39), and self-injurious behavior (40), and higher neuroticism (41). Consequently, it has been stated that the TAS-20 scale captures the core of alexithymic features adequately (36). Overall, it is necessary to keep in mind that there exist also other measures of alexithymia, for example, the Bermond-Vorst Alexithymia Questionnaire (BVAQ) that has been widely validated (42-45). 
Participants' socioeconomic factors included level of income and educational level. Educational level was classified into 3 categories (1=comprehensive school; $2=$ high school or occupational school; 3=academic level). Participants' level of income was assessed with a 13-point scale $(1=$ less than $5000 €$ per year; 13=more than $60000 €$ per year $)$.

\subsection{Statistical analyses}

Statistical analyses were conducted with STATA SE (version 13.0). Attrition refers to the drop-out of participants over follow-up measurements in longitudinal studies. The magnitude and potential biases of attrition can be investigated by comparing the included and excluded participants with regard to study variables. We investigated attrition by comparing the included and excluded participants in each study variable with independent samples t-test and chi-square tests of independence.

The association of somatic complaints with compassion was investigated using multilevel models for longitudinal design (growth curve models) with maximum likelihood estimation. In the analyses, the growth curve of compassion in 1997-2012 was set as outcome. Age was centered toward the age of 29 years (the age of the youngest participants at the first measurement point of compassion), in order to decrease multicollinearity in the analyses. We included in the multilevel models two types of effects: (1) fixed effects that refer to classic regression coefficients, and (2) random effects that refer to individual-level variation in the intercept, in the coefficient of age, and residual variance (i.e. withinindividual variation in the development of compassion over the follow-up). We predicted compassion by age, age-squared, somatic complaints (in 1986), and its age-interaction effects. Model 1 was adjusted for gender; model 2 also for use of health care due to somatic disease or physical accident and depression (in 1986), psychosocial family environment (stressful life events, parental alcohol intoxication, and parental care-giving) and socioeconomic family environment (in 1980); and 
participants' socioeconomic factors in adulthood (in 2011); and model 3 also for participants' alexithymic features (in 2011).

\section{Results}

The descriptive statistics of the study variables are shown in Table 1. Attrition analyses showed that women were more likely to participate than men $\left(32.6 \%\right.$ vs. $\left.24.9 \%, \chi^{2}(1)=26.34, p<.001\right)$. Included participants were older than excluded participants $(\mathrm{M}(35.8)$ vs. $\mathrm{M}(29.7), t(3594)=39.94, p<.001)$. Included participants had slightly higher level of compassion in $1997(\mathrm{M}(3.7)$ vs. $\mathrm{M}(3.5), t(2104)=5.20$, $p<.001)$, in $2001(\mathrm{M}(3.7)$ vs. $\mathrm{M}(3.6), t(2099)=3.07, p=.002)$, and in 2012 (M(3.8) vs. $\mathrm{M}(3.7)$, $t(1735)=3.67, p<.001)$. Furthermore, included participants were had higher score of stressful life events $(\mathrm{M}(0.6)$ vs. $\mathrm{M}(0.4), t(2877)=7.92, p<.001)$, lower level of parental alcohol intoxication $(\mathrm{M}(2.4)$ vs. $\mathrm{M}(2.6), t(3011)=-4.32, p<.001)$, less hostile parental care-giving style than excluded participants $(\mathrm{M}(0.3)$ vs. $\mathrm{M}(0.5), t(3596)=-5.36, p<.001)$, and a higher frequency of using health care $(23.9 \%$ vs. $\left.16.5 \%, \chi^{2}(1)=21.66, p<.001\right)$ than excluded participants. There was no attrition bias in the level of alexithymic features, depression, or the frequency of doctoral care due to accident. Regarding socioeconomic factors, included participants were less likely to have low educational level than excluded participants $\left(14.2 \%\right.$ vs. $\left.21.7 \%, \chi^{2}(1)=21.71, p<.001\right)$. Additionally, included participants' parents were less likely to have high educational level than excluded participants' parents (18.0\% vs. $\left.27.8 \%, \chi^{2}(1)=37.32, p<.001\right)$. There was no attrition bias in participants' or their parents' level of income. Previously, the missing values of psychosocial variables of the Young Finns data are shown to be missing at random (46). 
Table 1. The means, standard deviations (SD), frequencies, and ranges of the study variables.

\begin{tabular}{|c|c|c|c|c|}
\hline & Mean & $\mathrm{SD}$ & Measurement range & Frequency $(\%)$ \\
\hline Age (2001) & 35.8 & 2.4 & $34-40$ & \\
\hline Gender (female) & & & & $598(57.7)$ \\
\hline \multicolumn{5}{|l|}{ Parents' educational level (1980) } \\
\hline Comprehensive school & & & & $457(44.9)$ \\
\hline High school or occupational school & & & & $378(37.1)$ \\
\hline Academic level & & & & $183(18.0)$ \\
\hline Parents' level of income (1980) & 4.9 & 2.0 & $1-8$ & \\
\hline Stressful life events (1980) & 0.6 & 0.8 & $0-7$ & \\
\hline Parental alcohol intoxication (1980) & 2.4 & 1.4 & $1-8$ & \\
\hline Parental care-giving (1980) & 0.3 & 0.7 & $0-8$ & \\
\hline Number of somatic complaints (1986) & 1.6 & 1.6 & $0-10$ & \\
\hline Depression (1986) & 1.6 & 0.8 & $1-4$ & \\
\hline Used of health care (yes) (1986) & & & & $233(23.9)$ \\
\hline Doctoral care due to accident (yes) (1986) & & & & $300(30.0)$ \\
\hline \multicolumn{5}{|l|}{ Participants' educational level (2011) } \\
\hline Comprehensive school & & & & $130(14.2)$ \\
\hline High school or occupational school & & & & $537(58.8)$ \\
\hline Academic level & & & & $247(27.0)$ \\
\hline Participants' level of income (2011) & 7.3 & 3.0 & $1-13$ & \\
\hline Alexithymic features (2011) & 2.1 & 0.5 & $1-5$ & \\
\hline Compassion (1997) & 3.7 & 0.6 & $1-5$ & \\
\hline
\end{tabular}

Table 2 presents the results of the growth curve models. In any of the models, somatic complaints did not have a significant main effect on the developmental trajectory of compassion. This indicated that somatic complaints did not predict the level of compassion over the whole age range of the study. When adjusted only for gender, we obtained a significant positive interaction effect between compassion and age-squared $(\mathrm{B}=0.004, p=0.04)$. Additionally, in the fully-adjusted model, the significant interaction effects of compassion with age-squared remained $(\mathrm{B}=0.007, p=0.006)$ and a significant interaction with age also appeared $(\mathrm{B}=-0.137, p=0.02)$. The significant age-interaction effects indicated the effect of somatic complaints on compassion trajectory varied over age. The findings are illustrated in Figure 1. 
Somatic complaints and compassion

Table 2. Results of the multilevel models. Estimates (B) with $95 \%$ confidence intervals (CI) of compassion and age, when predicting standardized scores of compassion in adulthood. The intercept and random effects (residual variance, variance of intercept, and variance of age) were included in Models 1-3, but their estimates were excluded from this table for clarity.

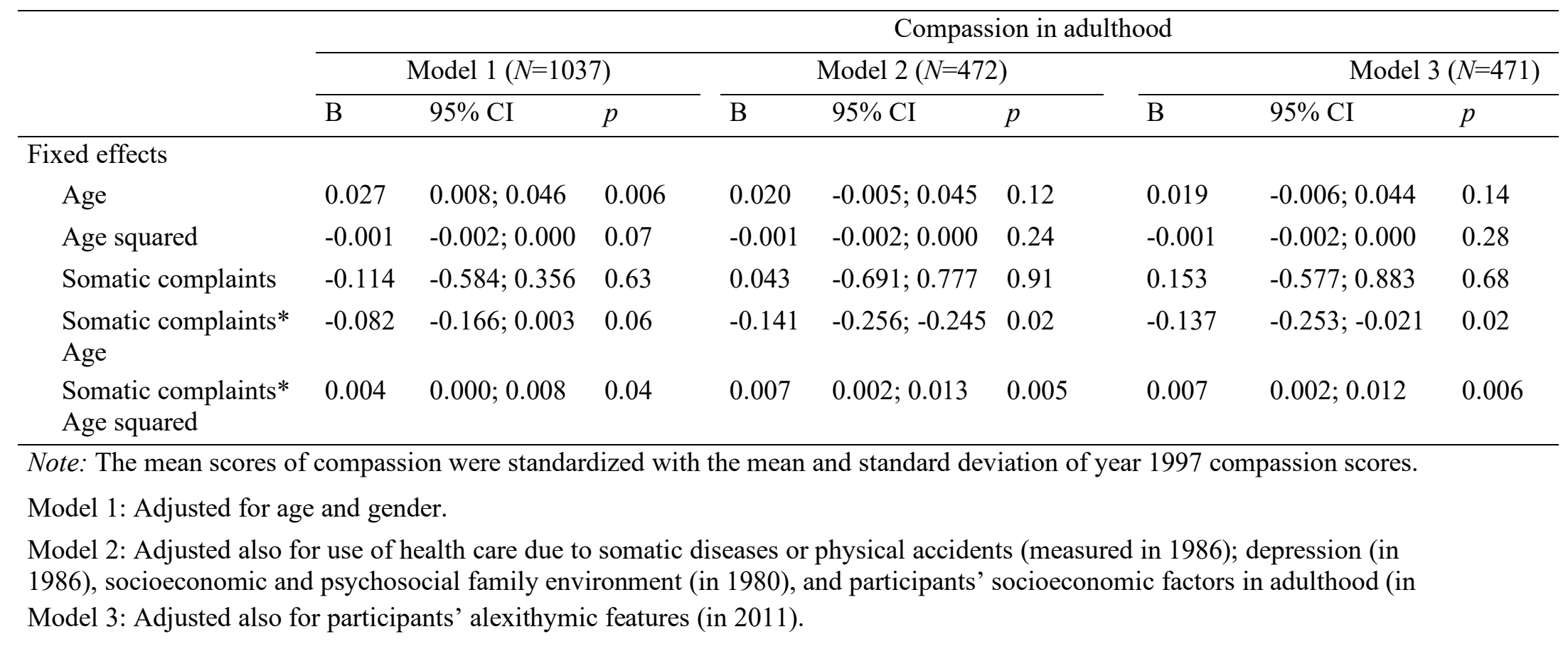




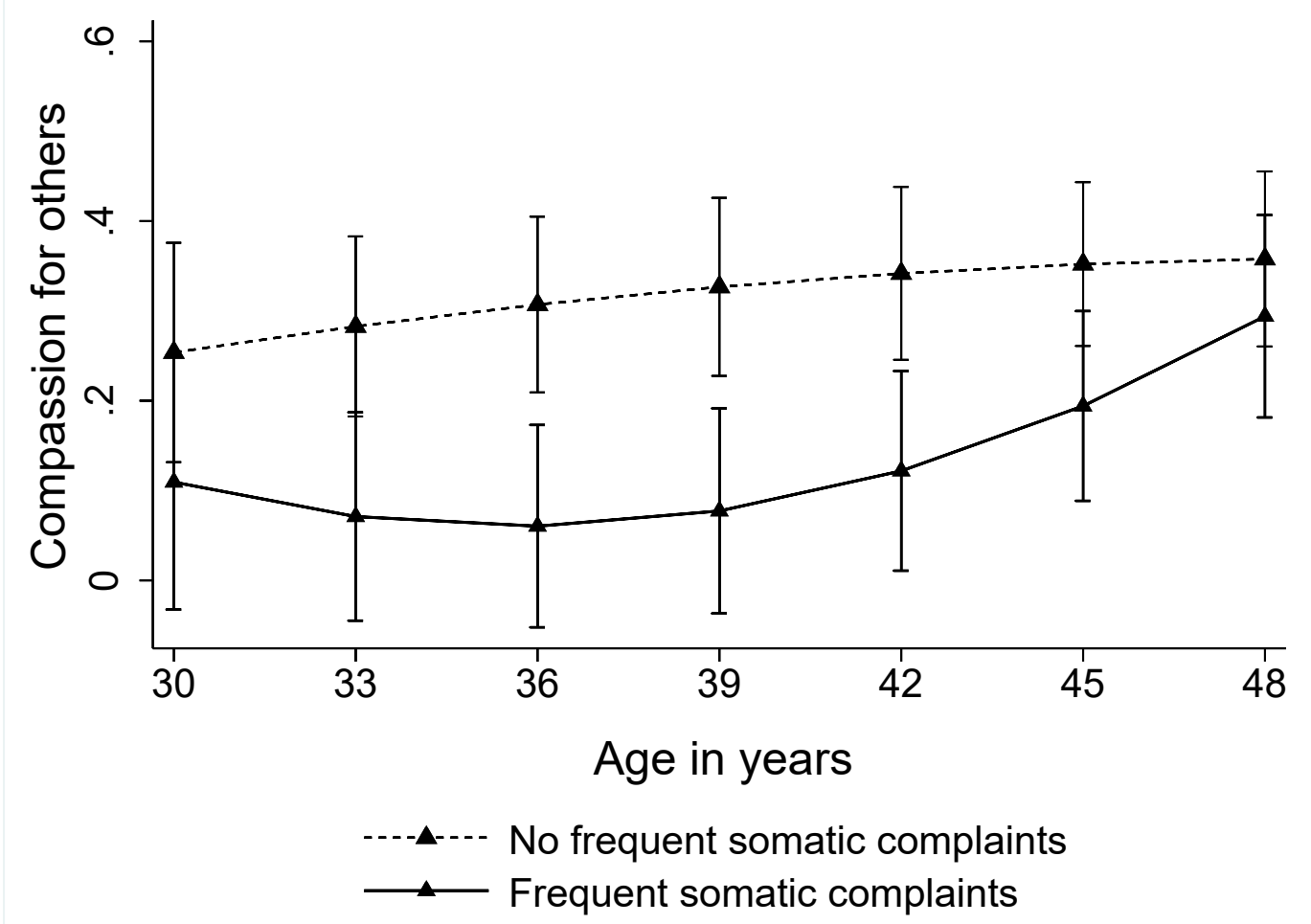

Figure 1. The trajectories of compassion development in adulthood separately for participants with frequent somatic complaints (highest $25 \%$ in the sample) and for others. Estimated means with $95 \%$ confidence intervals. Note: adjusted for age, gender, use of health care in childhood, depression in childhood, and childhood family environment (parental socioeconomic factors, parental care-giving, stressful life events, parental alcohol intoxication), and socioeconomic factors and alexithymic features in adulthood.

\section{Discussion}

To our knowledge, this study was the first to longitudinally investigate the relationship of somatic complaints with compassion. To our knowledge, this study was the first to longitudinally investigate the relationship of somatic complaints with compassion. Prior cross-sectional and family studies show that frequent somatic complaints are positively related to antisocial personality and alcohol use 
disorders in individuals cross-sectionally and in families $(7,8,16)$. Antisocial personality is characterized by low cooperativeness, including low empathy, compassion and moral reasoning $(17,18)$, so we hypothesized that somatic complaints would predict lower compassion. The results confirm this hypothesis: we found that frequent somatic complaints predicted less mature developmental trajectory of compassion in adulthood. In participants without frequent somatic complaints, compassion steadily increased over age in adulthood. In participants with frequent somatic complaints, however, compassion remained at a lower level until the age of 40 years, then started to increase, and achieved the normal level of compassion approximately at the age of 50 years. Taken together, frequent somatic complaints seem to predict lower compassion development in adulthood. The results also demonstrated that the association of somatic complaints with compassion development remained after controlling for participants' alexithymia.

Despite the enormous strain that somatic complaints set for the public health care system (3-5), very few of the individuals with frequent somatic complaints have received or feel need for psychiatric treatment in primary care (47). There is previous evidence for a substantial comorbidity of somatic complaints with psychiatric disorders, particularly with internalizing disorders such as anxiety, panic disorder, and depression $(20,27)$. Interestingly, our findings demonstrated that somatic complaints predict also less mature development of compassion. Low compassion, in turn, is strongly related to higher hostility and aggression toward others $(48,49)$, higher narcissistic traits $(50)$, and higher psychopathic features (51). Some of these features may have transmitted from parents to their offspring, since the parents of some somatizers are found to have higher incidence of criminality $(7,8)$. Taken together, our results tentatively suggest that there may exist a need for screening also hostility and externalizing symptomatology in individuals with frequent somatic complaints. However, there are complex patterns of relationship among different configurations of temperament and character with somatization, as described in detail elsewhere $(14,52,53)$. 
Somatic complaints and compassion

Previously, the co-occurrence of alexithymia with somatic complaints has been demonstrated (20-22). Alexithymia, however, is found not to reduce the effectiveness of treatments for patients with somatoform disorders (44). In line with this, our findings showed that some associations between somatic complaints and adverse developmental outcomes (i.e. low compassion) are independent of alexithymic features. Importantly, there is also evidence that alexithymia is related to a more limited range of coping strategies, for example, less frequent use of planful problem solving, cognitive reappraisal of the situation, or seeking for social support from close others (54). Accordingly, patients with fibromyalgia, for example, are found to benefit from adopting new coping strategies (55). Previously, high compassion is suggested to enhance stress coping as it predicts lower physiological stress levels when facing psychosocial stressors $(56,56)$. Hence, promoting compassion might also promote stress coping in somatizers.

There may exist, for example, reciprocal social pathways from somatic complaints to lower levels of compassion. It is a widely recognized challenge that individuals with somatic complaints (that are not explained by a medical condition) are commonly regarded as "difficult patients" by healthcare professionals and receive less understanding for their suffering from the physicians (27). Distressing meetings with healthcare professionals may further intensify somatization and increase the risk for the onset of comorbid psychiatric disorders. Our findings about the association between frequent somatic complaints and lower compassion arouse the question whether one complicating factor in patient-physician meetings might also be the patient's uncompassionate or hostile feelings, interpretations, or expectations toward the healthcare professionals. Hence, in some cases, there may exist a reciprocal vicious circle in the relationship between health care professionals and the patients with somatic complaints.

Previously, it has been found that somatic complaints are chronic or get worse over years only in $15-30 \%$ of the patients with medically unexplained somatic complaints (58). Interestingly, 
however, this study showed that the link between frequent somatic complaints and less mature compassion development can be obtained over a long-term follow-up in adulthood, after the somatic complaints have likely been alleviated. This may imply that frequent somatic complaints may be related to long-term alterations of the neurobiological systems that are also involved in compassion development. For example, cerebral opioidergic system is known to be involved both in the experiences of sensory pain (59) and social reward (60). Moreover, somatic complaints such as pain are found to correlate with the activation of amygdala, insula, nucleus accumbens, anterior cingulate, and inferior frontal cortex $(61,62)$. These brain regions are largely overlapping with the brain regions that correlate with empathy for pain (63) and display functional alterations after practicing compassion (6567). Taken together, there may exist also neurobiological pathways between somatic complaints and compassion.

Some limitations of the measures must be taken into consideration. Firstly, the questionnaire of somatic complaints has been developed for the Young Finns Study (YFS) by the medical YFS team. Hence, the validity of the questionnaire has not been examined in other datasets. Nevertheless, the internal consistency of the questionnaire was good. Furthermore, the questionnaire appeared to have good face validity since it included quite a comprehensive set of somatic symptoms (headache, stomachache, chest pain, backache, fatigue, exhaustion, dizziness, heartburn, heartbeat, or tension) that were evaluated with a 5-point scale. In the future, nevertheless, it is necessary to investigate the validity of the questionnaire in other datasets or to use other thoroughly validated questionnaires such as the Physical symptom checklist (PSC) (67) or the Patient Health Questionnaire (PHQ-15) (68), or to use a combination of several self-report questionnaires since it is shown to increase diagnostic accuracy (69).

Secondly, we evaluated the presence of participants' medical conditions with self-reports asking whether they had used health care services due to somatic diseases or physical accidents during 
the past six years. However, we did not use data from health care registers on participants' diagnoses of medical diseases. Hence, the precise origin of participants' somatic complaints cannot be surely confirmed. Current evidence states that somatic complaints may exist in the absence of any obtained medical condition; somatic complaints may be triggered by a medical condition but not directly result from the medical condition; or somatic complaints may intensify the physical symptoms related to a medical condition (1). It has been estimated that approximately $33 \%$ of individuals with somatic complaints do not have any currently recognized medical condition $(4,27)$. Overall, since there appears to be a reciprocal interplay between somatic complaints and medical conditions, the differentiation between somatic complaints and medical conditions would not be even possible.

Thirdly, our data did not provide possibilities to draw firm conclusions about the causal relationships between somatic complaints and compassion. That is, we could not control the baseline level of compassion at the measurement of somatic complaints. Theoretically, it is possible that compassion was at a lower level already before the onset of somatic complaints. Previously, it has been postulated that high compassion is related to better abilities to recognize the link between affective states and somatic complaints and, hence, to a lower risk for somatization symptoms $(70,71)$. Further, intervention studies have demonstrated that practicing compassion predicts lower cortisol levels and weaker inflammatory reactions when encountering stressful situations $(56,57)$. Low inflammatory reactions, in turn, are suggested to predict lower risk for somatic complaints (72). Nevertheless, due to the comparatively large population-based sample, very long follow-up with from early adulthood to middle age, and extensive set of covariates, our study provides strong support for the hypothesis that somatic complaints are associated with lower level of compassion.

With regard to clinical implications, there is evidence that cognitive-behavioral therapy (CBT) is an efficient treatment for patients with somatoform disorders $(73,74)$. Additionally, it has been proposed a mentalization-based approach that aims to, for example, enhance the recognition of cognitive- 
affective attachment patterns that may lead to elevated stress reactivity, to promote a unified approach to the mind and body, and to identify the links between interpersonal experiences and bodily sensations (75). Our findings suggest that there may also exist a need compassion-training exercises in patients with somatization symptoms. In compassion-training exercises, one focuses on how to identify somatic reactions deriving from different emotions and how to direct attentional focus from self-centered thoughts to other's affective processes (76). Additionally, compassion-training aims to increase abilities to find practical ways to reduce other's suffering and to recognize how one's prosocial acts toward others influence the arousing emotional states in one's own and other's mind (76).

\section{Acknowledgments}

This study was supported financially by the Academy of Finland (M.H., grant number 308676). The Young Finns Study has been financially supported by the Academy of Finland: Grants 322098, 286284, 134309 (Eye), 126925, 121584, 124282, 129378 (Salve), 117797 (Gendi), and 41071 (Skidi); the Social Insurance Institution of Finland; Competitive State Research Financing of the Expert Responsibility area of Kuopio, Tampere and Turku University Hospitals (grant X51001); the Juho Vainio Foundation; the Sigrid Juselius Foundation; the Yrjö Jahnsson Foundation; the Paavo Nurmi Foundation; the Finnish Foundation of Cardiovascular Research and Finnish Cultural Foundation; the Tampere Tuberculosis Foundation; the Emil Aaltonen Foundation; and Diabetes Research Foundation of Finnish Diabetes Association.

\section{Role of the funding source}

The funding source had no role in study design, data collection, data analysis, data interpretation, writing of the report, or in the decision to submit the article for publication. 


\section{Conflict of interest}

The authors declare that they have no conflict of interest.

\section{References}

(1) American Psychiatric Association (APA), Diagnostic and Statistical Manual of Mental Disorders (DSM-5®), American Psychiatric Pub, 2013.

(2) S. Sigvardsson, A.L. von Knorring, M. Bohman, C.R. Cloninger, An adoption study of somatoform disorders: I. The relationship of somatization to psychiatric disability, Archives of General Psychiatry 41 (1984) 853-859.

(3) W. Hiller, M.M. Fichter, W. Rief, A controlled treatment study of somatoform disorders including analysis of healthcare utilization and cost-effectiveness, J Psychosom Res 54 (2003) 369-380.

(4) S. Reid, S. Wessely, T.I.M. Crayford, M. Hotopf, Frequent attenders with medically unexplained symptoms: service use and costs in secondary care, Br J Psychiatry 180 (2002) 248-253.

(5) J.P.L. Haahr, P. Frost, J.H. Andersen, Predictors of health related job loss: a two-year follow-up study in a general working population, J Occup Rehabil 17 (2007) 581-592.

(6) R. Hoedeman, A.H. Blankenstein, B. Krol, P.C. Koopmans, J.W. Groothoff, The contribution of high levels of somatic symptom severity to sickness absence duration, disability and discharge, J Occup Rehabil 20 (2010) 264-273.

(7) M. Bohman, C.R. Cloninger, A.L. von Knorring, S. Sigvardsson, An adoption study of somatoform disorders: III. Cross-fostering analysis and genetic relationship to alcoholism and criminality, Archives of General Psychiatry 41 (1984) 872-878. 
(8) C.R. Cloninger, A.L. von Knorring, S. Sigvardsson, M. Bohman, P.M. Conneally, Symptom patterns and causes of somatization in men: II. Genetic and environmental independence from somatization in women, Genetic Epidemiology 3 (1986) 171-185.

(9) T.N. Hyphantis, T.C. Taunay, D.S. Macedo, M.G. Soeiro-de-Souza, L.W. Bisol, K.N. Fountoulakis, ... A.F. Carvalho, Affective temperaments and ego defense mechanisms associated with somatic symptom severity in a large sample, J Affect Disord 150 (2013) 481-489.

(10) L. Keltikangas-Järvinen, J. Kettunen, N. Ravaja, P. Näätänen, Inhibited and disinhibited temperament and autonomic stress reactivity, Int J Psychophysiol 33 (1999) 185-196.

(11) M. Ranger, M. Campbell-Yeo, Temperament and pain response: A review of the literature, Pain Manag Nurs 9 (2008) 2-9.

(12) F.H. Creed, I. Davies, J. Jackson, A. Littlewood, C. Chew-Graham, B. Tomenson, ... J. McBeth, The epidemiology of multiple somatic symptoms, J Psychosom Res 72 (2012) 311-317.

(13) F. Mai, Somatization disorder: a practical review, Can J Psychiatry 49 (2004) 652-662.

(14) I. Zwir, J. Arnedo, C. Del-Val, L. Pulkki-Råback, B. Konte, S.S. Yang,, .., C.R. Cloninger, Three genetic-environmental networks for human personality, Mol Psychiatry (2019) in press.

(15) E. Aksu, Y. Albayrak, E. Beyazyüz, N. Potas, F. Durankuş, B. Tenel, M. Beyazyüz, Distinct temperament and character traits in patients with hyperemesis gravidarum, Gynecol Endocrinol (2019) 1-5.

(16) S. Sigvardsson, A.L. von Knorring, M. Bohman, C.R. Cloninger, An adoption study of somatoform disorders. I. The relationship of somatization to psychiatric disability, Arch Gen Psychiatry 41 (1984) 853-859.

(17) C.R. Cloninger, D.M. Svrakic, T.R. Przybeck, A psychobiological model of temperament and character, Arch Gen Psychiatry 50 (1993) 975-990. 
(18) D.M. Svrakic, C. Whitehead, T.R. Przybeck, C.R. Cloninger, Differential diagnosis of personality disorders by the seven-factor model of temperament and character, Arch Gen Psychiatry 50 (1993) 991-999.

(19) J.L. Goetz, D. Keltner, E. Simon-Thomas, Compassion: an evolutionary analysis and empirical review, Psychol Bull 136 (2010) 351-374.

(20) F.N. Busch, Clinical approaches to somatization, J Clin Psychol 70 (2014) 419-427.

(21) V. Deary, T. Chalder, M. Sharpe, The cognitive behavioural model of medically unexplained symptoms: a theoretical and empirical review, Clin Psychol Rev 27 (2007) 781-797.

(22) A.K. Mattila, E. Kronholm, A. Jula, J.K. Salminen, A.M. Koivisto, R.L. Mielonen, M. Joukamaa, Alexithymia and somatization in general population, Psychosom Med 70 (2008) 716-722.

(23) R.M. Bagby, L.C. Quilty, G.J. Taylor, H.J. Grabe, O. Luminet, R. Verissimo, et al., Are there subtypes of alexithymia? Pers Individ Dif 47 (2009) 413-8. doi:10.1016/j.paid.2009.04.012

(24) H.C. Vorst, B. Bermond, Validity and reliability of the Bermond-Vorst alexithymia questionnaire, Personality and Individual Differences 30 (2001) 413-434.

(25) D. Samur, M. Tops, C. Schlinkert, M. Quirin, P. Cuijpers, S.L. Koole, Four decades of research on alexithymia: moving toward clinical applications, Front Psychol 4 (2013) 861.

(26) J. Crocker, M.A. Olivier, N. Nuer, Self-image goals and compassionate goals: Costs and benefits, Self and Identity 8 (2009) 251-269.

(27) K. Kroenke, Patients presenting with somatic complaints: epidemiology, psychiatric co-morbidity and management, Int J Methods Psychiatr Res 12 (2003) 34-43.

(28) P.R. Steffen, K.S. Masters, Does compassion mediate the intrinsic religion-health relationship, Ann Behav Med 30 (2005) 217-224. 
(29) O.T. Raitakari, M. Juonala, T. Rönnemaa, L. Keltikangas-Järvinen, L. Räsänen, M. Pietikäinen, ..., A. Jula, Cohort profile: the cardiovascular risk in Young Finns Study, Int J Epidemiol 37 (2008) $1220-1226$.

(30) C.R. Cloninger, T.R. Przybeck, D.M. Švrakić, The Temperament and Character Inventory (TCI): A guide to its development and use. St. Louis, MO: Center for Psychobiology of Personality, Washington, 1994.

(31) A.I. Saarinen, L. Keltikangas-Järvinen, L. Pulkki-Råback, C.R. Cloninger, M. Elovainio, T. Lehtimäki, ..., M. Hintsanen, The relationship of dispositional compassion with well-being: a study with a 15-year prospective follow-up, J Posit Psychol (2019) 1-15.

(32) K. Josefsson, M. Jokela, M. Hintsanen, C.R. Cloninger, L. Pulkki-Råback, P. Merjonen, .... L. Keltikangas-Järvinen, Parental care-giving and home environment predicting offspring's temperament and character traits after 18 years, Psychiatry Res 209 (2013) 643-651.

(33) R.M. Bagby, G.J. Taylor, J.D. Parker, The twenty-item Toronto Alexithymia Scale-II. Convergent, discriminant, and concurrent validity, J Psychosom Res 38 (1994) 33-40.

(34) M. Karukivi, A. Jula, N. Hutri-Kähönen, M. Juonala, O. Raitakari, Is alexithymia associated with metabolic syndrome? A study in a healthy adult population, Psychiatry Res 236 (2016) 58-63.

(35) J. Wessman, S. Schönauer, J. Miettunen, H. Turunen, P. Parviainen, J.K. Seppänen, et al., Temperament clusters in a normal population: implications for health and disease. PloS One 7 (2012) e33088.

(36) G. Craparo, P. Faraci, A. Gori, Psychometric properties of the 20-item Toronto Alexithymia Scale in a group of Italian younger adolescents, Psychiatry Investig 12 (2015) 500-507.

(37) G.J. Taylor, R.M. Bagby, J.D. Parker, The 20-Item Toronto Alexithymia Scale: IV. Reliability and factorial validity in different languages and cultures, Journal of Psychosomatic Research 55 (2003) 277-283. 
(38) K. Sayar, S. Kose, H.J. Grabe, M. Topbas, Alexithymia and dissociative tendencies in an adolescent sample from Eastern Turkey, Psychiatry and Clinical Neurosciences 59 (2005) 127-134.

(39) K.L. Ledouc, Alexithymia, trauma and posttraumatic stress disorder in incarcerated juvenile offenders, Diss Abstr Int Sect B Sci Eng 63 (2002) 1036.

(40) S.C. Paivio, C.R. McCulloch, Alexithymia as a mediator between childhood trauma and selfinjurious behaviors, Child Abuse \& Neglect 28 (2004) 339-354.

(41) R.M. Bagby, L.E. Ayearst, R.A. Morariu, C. Watters, G.J. Taylor, The internet administration version of the 20-item Toronto Alexithymia Scale, Psychol Assess 26 (2014) 16.

(42) B. Bermond, K. Clayton, A. Liberova, O. Luminet, T. Maruszewski, P.E. Ricci Bitti, et al., A cognitive and an affective dimension of alexithymia in six languages and seven populations, Cogn Emot 21 (2007) 1125-36.

(43) A.-S. Deborde, S. Berthoz, J. Wallier, J. Fermanian, B. Falissard, P. Jeammet, et al., The BermondVorst Alexithymia Questionnaire cutoff scores: a study in eating-disordered and control subjects, Psychopathology 41 (2007) 43-9.

(44) L. de Vroege L, W.H.M. Emons, K. Sijtsma, C.M. van der Feltz-Cornelis, Alexithymia Has No Clinically Relevant Association With Outcome of Multimodal Treatment Tailored to Needs of Patients Suffering From Somatic Symptom and Related Disorders, A Clinical Prospective Study, Front. Psychiatry 9 (2018)) 292.

(45) R. Hornsveld, F. Kraaimaat, Alexithymia in Dutch violent forensic psychiatric outpatients. Psychol Crime Law 18 (2012) 833-46. doi:10.1080/1068316X. 2011.568416

(46) L. Pulkki-Råback, M. Elovainio, C. Hakulinen, J. Lipsanen, M. Hintsanen, M. Jokela, ..., L. Keltikangas-Järvinen, Cumulative effect of psychosocial factors in youth on ideal cardiovascular health in adulthood: the Cardiovascular Risk in Young Finns Study, Circulation 131 (2015) 245-253. 
(47) H. Karlsson, V. Lehtinen, M. Joukamaa, Psychiatric morbidity among frequent attender patients in primary care, General Hosp Psychiatry 17 (1995) 19-25.

(48) Ó. García, A. Aluja, L.F. García, S. Escorial, A. Blanch, Zuckerman-Kuhlman-Aluja Personality Questionnaire (ZKA-PQ) and Cloninger's Temperament and Character Inventory Revised (TCI-R): A comparative study, Scand J Psychol 53 (2012) 247-257.

(49) J. Lee, S.K. Min, K.-H. Kim, B. Kim, S.J. Cho, S.-H. Lee, ..., S.-Y. Suh, Differences in temperament and character dimensions of personality between patients with Hwa-byung, an anger syndrome, and patients with major depressive disorder, J Affect Disord 138 (2012) 110-116.

(50) F. De Fruyt, B.J. De Clercq, L. van de Wiele, K. Van Heeringen, The validity of Cloninger's Psychobiological Model versus the Five-Factor Model to predict DSM-IV personality disorders in a heterogeneous psychiatric sample: Domain facet and residualized descriptions, Journal of Personality 74 (2006) 479-510.

(51) T. Paal, T. Bereczkei, Adult theory of mind, cooperation, Machiavellianism: The effect of mindreading on social relations, Pers Individ Dif 43 (2007) 541-551.

(52) I. Zwir, J. Arnedo, C. Del-Val, L. Pulkki-Råback, B. Konte, S.S. Yang, .., C.R. Cloninger, Uncovering the complex genetics of human character, Mol Psychiatry (2018a).

(53) I. Zwir, J. Arnedo, C. Del-Val, L. Pulkki-Råback, B. Konte, S.S. Yang, .., C.R. Cloninger, Uncovering the complex genetics of human temperament, Mol Psychiatry (2018b).

(54) T. Tominaga, H. Choi, Y. Nagoshi, Y. Wada, K. Fukui, Relationship between alexithymia and coping strategies in patients with somatoform disorder, Neuropsychiatr Dis Treat 10 (2014) 55-62.

(55) R. Geenen, L. van Ooijen-van der Linden, M.A. Lumley, J.W. Bijlsma, H. van Middendorp, The match-mismatch model of emotion processing styles and emotion regulation strategies in fibromyalgia, J Psychosomat Res. 72 (2012) 45-50. 
(56) J.L. Abelson, T.M. Erickson, S.E. Mayer, J. Crocker, H. Briggs, N.L. Lopez-Duran, I. Liberzon, Brief cognitive intervention can modulate neuroendocrine stress responses to the Trier Social Stress Test: Buffering effects of a compassionate goal orientation, Psychoneuroendocrinology 44 (2014) $60-70$.

(57) T.W. Pace, L.T. Negi, D.D. Adame, S.P. Cole, T.I. Sivilli, T.D. Brown, ..., C.L. Raison, Effect of compassion meditation on neuroendocrine, innate immune and behavioral responses to psychosocial stress, Psychoneuroendocrinology 34 (2009) 87-98.

(58) T.C. olde Hartman, M.S. Borghuis, P.L. Lucassen, F.A. van de Laar, A.E. Speckens, C. van Weel, Medically unexplained symptoms, somatisation disorder and hypochondriasis: course and prognosis. A systematic review, J Psychosom Res 66 (2009) 363-377.

(59) J.K. Zubieta, Y.R. Smith, J.A. Bueller, Y. Xu, M.R. Kilbourn, D.M. Jewett, ..., C.S. Stohler, Regional mu opioid receptor regulation of sensory and affective dimensions of pain, Science 293 (2001) 311-315.

(60) J. Le Merrer, J.A. Becker, K. Befort, B.L. Kieffer, Reward processing by the opioid system in the brain, Physiol Rev 89 (2009) 1379-1412.

(61) U. Bingel, M. Quante, R. Knab, B. Bromm, C. Weiller, C. Büchel, Subcortical structures involved in pain processing: evidence from single-trial fMRI, Pain 99 (2002) 313-321.

(62) K. Bornhövd, M. Quante, V. Glauche, B. Bromm, C. Weiller, C. Büchel, Painful stimuli evoke different stimulus-response functions in the amygdala, prefrontal, insula and somatosensory cortex: a single-trial fMRI study, Brain 125 (2002) 1326-1336.

(63) C. Lamm, J. Decety, T. Singer, Meta-analytic evidence for common and distinct neural networks associated with directly experienced pain and empathy for pain, Neuroimage 54 (2011) 2492-2502. 
(64) O.M. Klimecki, S. Leiberg, M. Ricard, T. Singer, Differential pattern of functional brain plasticity after compassion and empathy training, Soc Cogn and Affect Neurosci 9 (2013) 873-879.

(65) H.Y. Weng, A.S. Fox, A.J. Shackman, D.E. Stodola, J.Z. Caldwell, M.C. Olson, ..., R.J. Davidson, Compassion training alters altruism and neural responses to suffering, Psychol Sci 24 (2013) 1171-1180.

(66) H.Y. Weng, R.C. Lapate, D.E. Stodola, G.M. Rogers, R.J. Davidson, Visual attention to suffering after compassion training is associated with decreased amygdala responses, Front Psychol (2018) 9.

(67) L. de Vroege, W.H.M. Emons, K. Sijtsma, C.M. van der Feltz-Cornelis, Alexithymia Has No Clinically Relevant Association With Outcome of Multimodal Treatment Tailored to Needs of Patients Suffering From Somatic Symptom and Related Disorders, A Clinical Prospective Study, Front. Psychiatry 9 (2018b) 292.

(68) K. Kroenke, R.L. Spitzer, J.B. Williams, The PHQ-15: validity of a new measure for evaluating the severity of somatic symptoms, Psychosom Med 64 (2002) 258-266.

(69) J.A. Laferton, N.M. Stenzel, W. Rief, K. Klaus, E. Brähler, R. Mewes, Screening for DSM-5 somatic symptom disorder: diagnostic accuracy of self-report measures within a population sample, Psychosom Med 79 (2017) 974-981.

(70) D.D. Clark, Diagnosis and treatment of medically unexplained symptoms and chronic functional syndromes, Fam Syst Health 34 (2016) 309-316.

(71) C.R. Cloninger, M. Dokucu, Somatizing and Dissociative Disorders. In The Medical Basis of Psychiatry (pp. 195-209). Springer, New York, NY, 2016.

(72) M. Maes, Inflammatory and oxidative and nitrosative stress pathways underpinning chronic fatigue, somatization and psychosomatic symptoms, Current Opinion in Psychiatry 22 (2009) 75-83. 
(73) L.A. Allen, R.L. Woolfolk, Cognitive behavioral therapy for somatoform disorders, Psychiatric Clin North Am 33 (2010) 579-593.

(74) K. Kroenke, Efficacy of treatment for somatoform disorder: a review of randomized controlled trials, J Psychosom Med 69 (2007) 881-888.

(75) P. Luyten, B. Van Houdenhove, A. Lemma, M. Target, P. Fonagy, A mentalization-based approach to the understanding and treatment of functional somatic disorders, Psychoanalyt Psychother 26 (2012) $121-40$.

(76) J. Kirby, P. Gilbert,. The emergence of the Compassion Focused Therapies. In: P. Gilbert (Ed). Compassion: Concepts, Research and Applications (2017) (p. 258-285). London: Routledge. 
Supplementary Table 1 . The study design.

\begin{tabular}{|c|c|c|c|c|c|c|}
\hline & 1980 & 1986 & 1997 & 2001 & 2011 & 2012 \\
\hline Parental socioeconomic factors & $\mathrm{X}$ & & & & & \\
\hline Stressful life events & $\mathrm{X}$ & & & & & \\
\hline Parental care-giving & $\mathrm{X}$ & & & & & \\
\hline Parental alcohol intoxication & $\mathrm{X}$ & & & & & \\
\hline Somatic complaints & & $\mathrm{X}$ & & & & \\
\hline Depression & & $\mathrm{X}$ & & & & \\
\hline Use of health care & & $\mathrm{X}$ & & & & \\
\hline Participant's socioeconomic factors & & & & & $\mathrm{X}$ & \\
\hline Alexithymic features & & & & & $\mathrm{X}$ & \\
\hline Compassion & & & $\mathrm{X}$ & $\mathrm{X}$ & & $\mathrm{X}$ \\
\hline
\end{tabular}

